\title{
Cave Lighting Control System Based on IOT Location*
}

\author{
Deng Jian-zhi, Cheng Xiao-hui \\ College of Information Science and Engineering GuiLin University of Technology, GuiLin 541004, China \\ dengjianzhi@163.com, cxiaohui@tom.com
}

\begin{abstract}
A cave lighting control system based on Internet of Things location is designed in this paper. The design is a sub-system of the IOT electronic guiding system based on Zigbee. Structure, principle, software, hardware and network are introduced in the paper. CC2431 is used to be a node when CC2430 is used to be a router. CC2431 can know its location from the location information and RSSI of CC2430, and control the light in the cave.

Index Terms - Internet Of Things; location; cave; lighting
\end{abstract} control

\section{Introduction}

GuiLin is the famous tourist city in the world. It has acknowledged the karst topography. There are more than 20 national "3A level" scenic spots in GuiLin city. Cave tourist is one kind of tourist ways to see the strange stones in the cave. In order to show the stones, the lighting effects are the best and also the only way in the dark sight. So how to place and control the lighting is very important.

There are two parts of the cave lighting control. One part is used to show the road and route. The other part is used to show the views. In the traditional way, the tourists made a team and followed the guide when they traveled. The guide introduced the views and turn-on or off the lights. Now, more and more people like to take a personal travel. Because they don't need to play follow a strict travel schedule just like doing a work. They can go, stay and take photos any where, any time. But in this way, all the lights in the cave would be turned on all the time. It's a waste when there are few people in the cave. So we need a smart guide system that can show the views and also control the lights.[1]

In this paper, a cave lighting control system based on Internet of Things location is designed to solve the problem. It's a sub-system of the smart guide system that we designed.

\section{System Design}

\section{A. System Structure}

The system designed in this paper is a sub-system of IOT position smart guide system. The smart guide system is made up of smart guide PDA and wireless position and control network. The PDA would be carried by the tourist. It is a PDA setup our tourist APP. The PDA is made up of MCU, LCD, radio, power and wireless module. The wireless network is made up of server, coordinator, router, node and lighting control module.
The lighting control system is a network based on Zigbee, an IEEE 802 standard personal area networks that work in $2.4 \mathrm{GHz}$. It is a protocol using small, low-power digital radios. ZigBee networks can be formed ad-hoc, with no centralized control or high-power transmitter/receiver able to reach all of the devices. Any ZigBee device can be tasked with running the network.

\section{B. Principle of System}

When the tourist comes into the cave with a smart guide PDA that setup a Zigbee position module, he also comes into the Zigbee position network. The PDA will enter the network and communicate with the router and locate itself. Then, it can turn on the lights in front and turn off the light behind.

When the tourist goes into a scenic area, the system can also turn on the scenic lights. And turn off them when the tourist goes to next scenic area.

The location information in the system is on the basis of the RSSI(Received Signal Strength Indicator). The distance between two nodes can be judged according to the RSSI value that the node detected.

Fig 1 is a model used to detect the position. The 4 white circles are the location reference nodes. The reference nodes setup in the position that had already known. So they can provide their position information when other node need. The black circle is so-called a blind node. Its position is unknown. There is a hardware location engine in the node. The blind node can locate itself from the RSSI value that the reference nodes provided. [2 6]

\section{Hardware Design}

According to the Zigbee protocol, the hardware of the lighting control system is made up of node, router and coordinator.

1) node: the smart guide PDA is the blind node in the Zigbee network. S3C2440 is used to make the MCU of the PDA. And CC2431 is the wireless module. The S3C2440 is a 16/32-bit RISC microprocessor developed with ARM920T core. It implements MMU, AMBA BUS, and Harvard cache architecture. And it's designed to use in hand-held devices and general applications with low-power, and high-performance. WinCE, Linux, and Andriod can easily be transplanted on the PDA.

\footnotetext{
* This work is partially supported by National Natural Science Funded Project (No: 61262075) and Guangxi College Funded Project (No: 201201ZD012).
} 


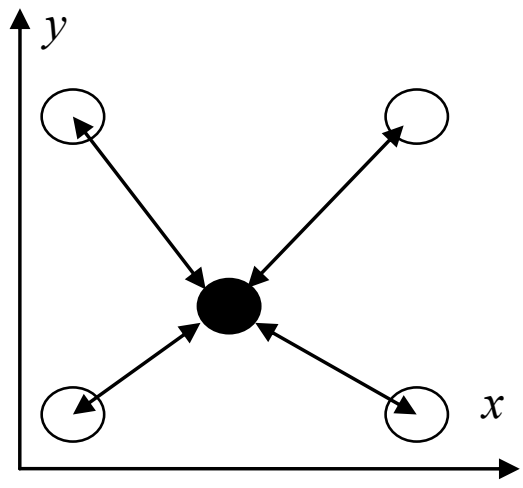

Fig 1 Principle of location

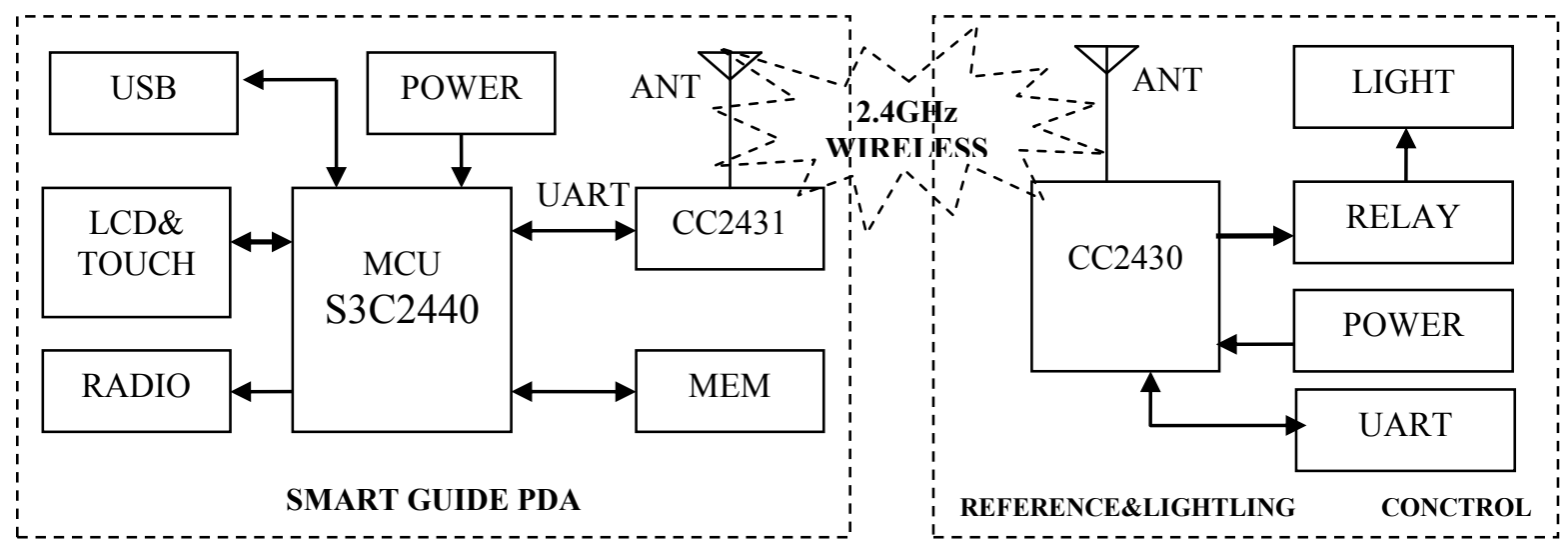

Fig 2 Schematic diagram of the system

2) router: the router is the location reference node, and it's also used to control the lights. The CC2430 is used to make the router. The CC2430 is a CC2431 without location engine. There are more than $20 \mathrm{I} / \mathrm{Os}$ in CC2430. The lights are controlled by the relays that connected to CC2430. [8]

3) coordinator: coordinator is used to manage the Zigbee network and connected with server.

\section{System Arrangement}

\section{A. Router arrangement of system}

A blind node can know the location from at least 3 reference nodes. We know from the test in lab that 2 nodes can still communicate in $30-50 \mathrm{~m}$. And the blind node can be located in about $2 \mathrm{~m}$ area.

As we all know, the cave is full of stone. Structure of karst topography cave is complex. Because of the special situation of the cave, the nodes arranged in the cave are followed the rules:

1) The routers are arranged on the two sides of the road;

2) The distance $d$ between 2 routers on one side is on more than $40 \mathrm{~m}$;

3) The distance $s$ between 2 routers on two sides is on more than $20 \mathrm{~m}$;

4) Put a router on the corner or obstacle;
5) A router controls the light ahead and at the back.

\section{node}

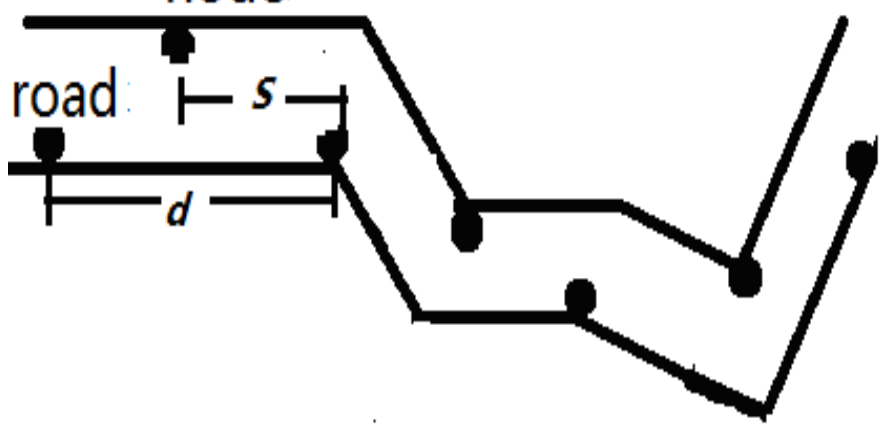

Fig 3 Router arrangement of system

B. Partition of the location area and the lighting control area

Because the lights are controlled by the routers, the location areas are separated into some lighting control area. For example, the location areas are separated into some triangle areas. The lights in a lighting control area are control by the same relays.

Fig 4 is a module of Partition of the location area and the lighting control area. Area I, II, III and IV are 4 triangle areas 
that encircle with 3 closest routers. The areas are separated into some sub-areas. Each sub-area is a lighting area that control by a router.

When a tourist goes straight the road in fig 4, the lights will be turned on and off from area I2+II1 to II2+III1, II3+III2, III3+IV1 and IV2 in turn.

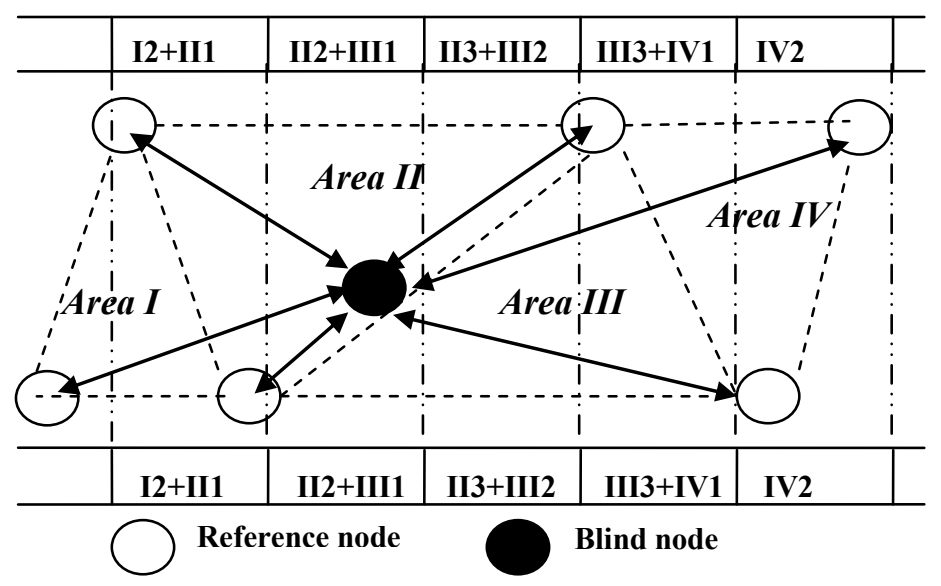

Fig 4 Partition of the location area and the lighting control area

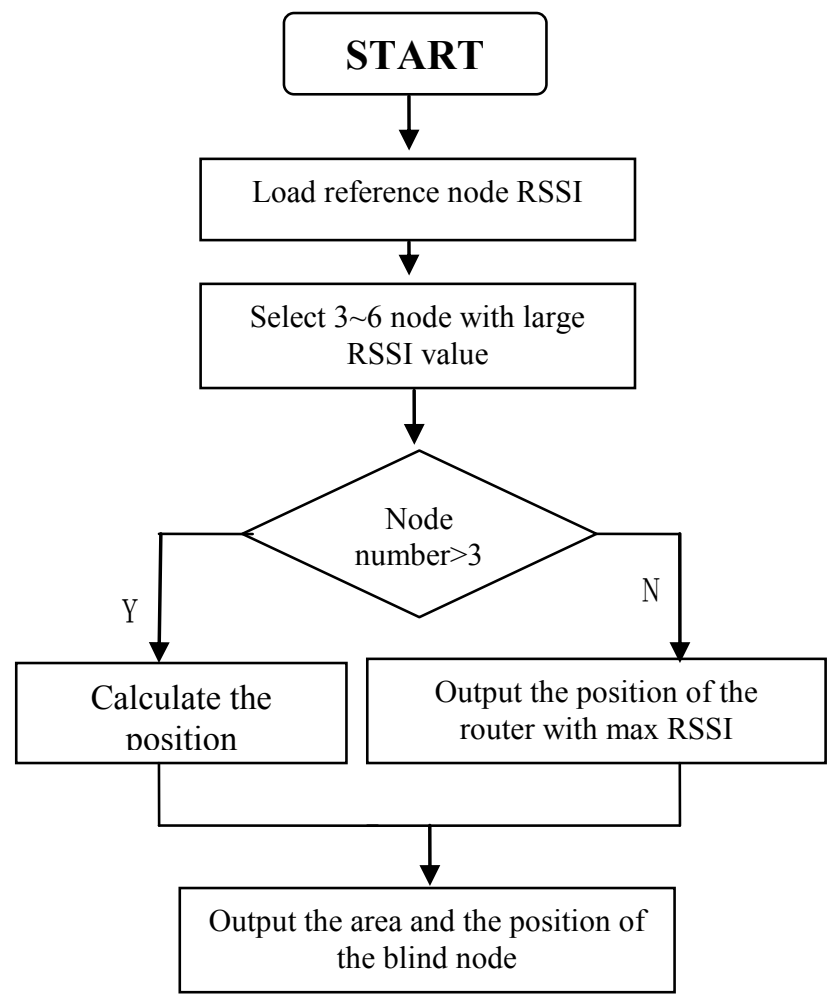

Fig 5 Algorithm of location

\section{System Software}

\section{A. Location Algorithm}

The location information is used in 2 purposes. One is to control the lights. The other one is to show the view in video on PDA automatically. The algorithm of location is showed in Fig 5.

\section{B. Position inquire and lighting control Algorithm}

When the tourist comes into the cave with a smart guide PDA, the PDA will enter the Zigbee network. The PDA sends position inquiry to the references. The reference nodes accept and response the position and RSSI information. CC2431 calculates location and send its position to the routers. The routers turn on the light according to the position.

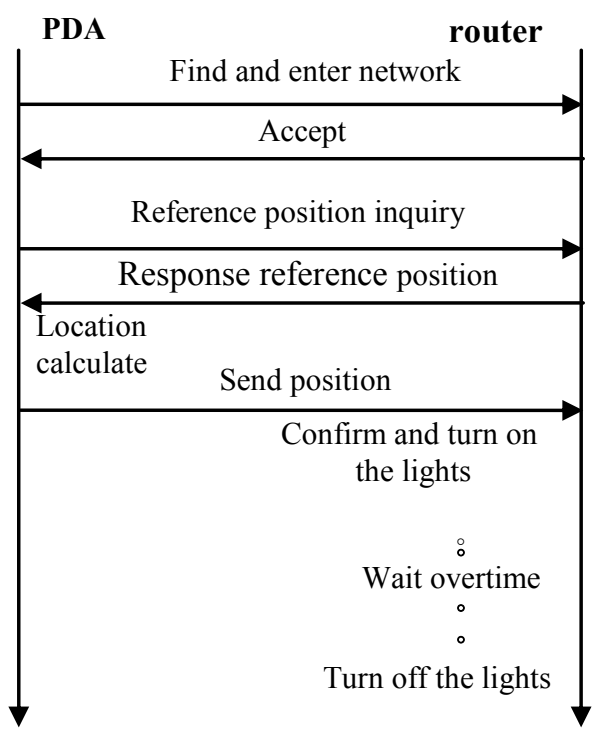

Fig 6 Diagram location and lighting control

The router waits for an inquiry from PDA, and turn off the light if waiting overtime.

\section{Operating System(OS)}

The OS of the lighting control system is TinyOS. TinyOS is a free, open source, component-based operating system targeting wireless sensor network(WSN). TinyOS is built out of components. Basic components, uart components, location components, and I/O components are transplanted.[8,9]

\section{Conclusion}

In this paper, a cave lighting control system based on Internet of Things location is designed. The system structure, location and control principle, software, hardware designs are described. It's a sub-system of the IOT electronic guiding system. The smart guide PDA locates itself by calculating the position and RSSI.

\section{Acknowledgment}

The authors thank the sponsorships from National Natural Science Funded Project (No: 61262075), and GuangXi College Funded Project (No: 201201ZD012). We would like to extend our gratitude to them. 


\section{References}

[1] Deng Jian-zhi, Cheng Xiao-hui. The Design of Video and Audio Cave Electronic Guiding System Based on UHF RFID. Video Engineering. vol.36,no.19.pp.89-92,October.2012

[2] Liang Hao, Chen Xinrong. Solar cell monitoring system based on wireless sensor network. Electric Power Automation Equipment. 2010.9:125-128

[3] Li Zhanbo, Liu Huiling. Mobile wireless sensor network nod localization based on Zigbee. Computer Engineering And Design. 2012.2: 431-434

[4] Vinod Kulathumani, Anish Arora, and Sricharan Ramagiri. Pursuit Control Over Wireless Sensor Networks Using Distance Sensitivity Properties. IEEE Transaction on Automatic Control, 2011,10: 2473-2479
[5] Rafael Falcon, Xu Li, Amiya Nayak. Carrier-Based Focused Coverage Formation in Wireless Sensor and Robot Networks. IEEE Transaction on Automatic Control, 2011,10:2406-2410

[6] Feng Jun, Ning Zhigang, Yang Puqiong. Design of wireless meter reading system based on ZigBee. Electric Power Automation Equipment. 2010.8:108-112

[7] CC2431 location engineering [EB/OL]. 2006

[8] CC2340 datasheet[EB/OL].2007

[9] Philip Levis and David Gay. TinyOS Programming [EB/OL]. 2009,7

[10] Cheng Long, Yang Bo. Transplantation of Embedded Operating System TinyOS for Wireless Sensor Network Nodes. Computer Science. 2011.1:323-325 